\title{
Mode Stabilization Mechanism of Buried-Waveguide Lasers with Lateral Diffused Junctions
}

\author{
K. L. YU, KAM Y. LAU, MEMBER, IEEE, SHLOMO MARGALIT, UZIEL KOREN, \\ T. R. CHEN, AND AMNON YARIV, FELLOW, IEEE
}

\begin{abstract}
The mode stabilization behavior of the buried active waveguide with lateral diffused junction is theoretically investigated. The study shows that for an active waveguide of width around $5 \mu \mathrm{m}$ with a lateral diffused junction in the middle, the single fundamental transverse mode is preferred as the injection level is raised. The theoretical results are found to be in good agreement with experimental results observed in the groove transverse junction $\mathrm{InGaAsP}_{\mathbf{n}} \mathrm{InP}$ laser.
\end{abstract}

\section{INTRODUCTION}

$\mathrm{T}$ HE transverse modal control of semiconductor injection lasers is a subject of active research. Applications of semiconductor lasers, for long-haul fiber-optics communication, video-disk recording and readout, laser printing, etc., require high-power operation of injection lasers combined with a stable single mode. Hence, it is important to understand the mechanisms which affect the transverse modal behavior, especially under high-power operation.

In strongly guiding dielectric waveguide lasers which can support several modes, such as the buried heterostructure (BH) or the channeled substrate $\mathrm{BH}$ lasers, it is very difficult to obtain fundamental mode operation when the waveguide width is larger than $\sim 2 \mu \mathrm{m}$ [1]. For vertical injection (i.e., the $\mathrm{p}-\mathrm{n}$ junction is parallel to the wide dimension of the waveguide), spatial hole burning gives rise to a dip in the gain profile at the center of the waveguide, which leads to preferred lasing at higher order modes. Some discrimination in favor of the fundamental mode can be provided by a very narrow stripe contact located centrally above the waveguide.

In a recent work [2], we have demonstrated experimentally that in a wide (3-5 $\mu \mathrm{m})$ buried waveguide with a transverse junction type of injection (i.e., with a transverse p-n junction located near the middle of the waveguide), the gain profile favors lasing in the fundamental mode. In the following analysis we will show that as the optical power increases, the gain profile becomes progressively narrower due to the decreased stimulated lifetime, and thus the effective carrier diffusion length. If the transverse junction is situated near the center of the waveguide, this effect provides stabilization for the fundamental mode by increasing its gain relative to that of higher order modes. For simplicity, the analysis is

Manuscript received May 14, 1982; revised August 23, 1982.

K. L. Yu, S. Margalit, U. Koren, and A. Yariv are with the California Institute of Technology, Pasadena, CA 91125.

K. Y. Lau is with the Ortel Corporation, Alhambra, CA 91803.

T. R. Chen is with the California Institute of Technology, Pasadena, CA 91125 , on leave from the Chengdu Institute of Radio Engineering, People's Republic of China. carried out on buried rectangular waveguide with a lateral diffused junction. Since, as will be shown below, the lateral mode stabilization results mainly from the lateral diffusion of injected carriers, the results of the analysis can be applied to waveguides of other shapes. Results of the analysis will be compared with experimental observations of the transverse modal behavior of a grooved TJS laser with a crescent-shaped active region. Similar approaches had been carried out on broad area P-p-n-N GaAs-GaAlAs vertical structures [3]-[7], in an effort to stabilize the transverse mode pattern perpendicular to the junction plane. The thickness of the optical cavity layer in those structures was limited to $\sim 2 \mu \mathrm{m}$, and thus uniform gain had been assumed. In contrast, the effect of diffusion of injected carriers on the gain is very significant in the present analysis.

\section{Model of the Gain Stabilized Waveguide}

In this work, we study in detail the gain stabilizing mechanism for a laser with a dielectric slab waveguide structure shown in Fig. 1. The width of the waveguide is $2 W$, and the junction is located at $x=a$ where $-W \leqslant a \leqslant W$. Since guiding is due to a real-index profile, we take the optical transverse mode intensity profile $S(x)$ as independent of the injection level:

$$
\begin{array}{rlrl}
S(x) & =S_{0} \sin ^{2} \frac{\pi(m+1)(x+b)}{2 b} & |x| \leqslant b \\
S(x) & =0 & |x| \geqslant b \\
b=W-\xi & \xi / W \ll 1
\end{array}
$$

where $S_{0} b$ is the total optical power in the mode, and $m=$ $0,1,2,3, \cdots$ is the mode order. The geometrical shape of the crescent waveguide is accounted for by taking positive values of $\xi$. The steady state lateral distribution of the photon density and the excess carrier densities inside the active region are described by the following equations:

$$
\begin{aligned}
& D_{\mathrm{eff}} \frac{d^{2} \Delta(x)}{d x^{2}}=\frac{\Delta(x)}{\tau_{s}}+A\left(\Delta(x)-n_{o m}\right) S(x) \\
& \frac{\int_{-W}^{+W} S(x) d x}{\tau_{p h}}=\int_{-W}^{+W} A\left(\Delta(x)-n_{o m}\right) S(x) d x
\end{aligned}
$$

where $\Delta(x)$ denotes the excess carrier densities, $\Delta_{n}$ on the $n$ side, and $\Delta_{p}$ on the $p$ side. In (3) and (4), $D_{\text {eff }}(x)$ is the diffu- 


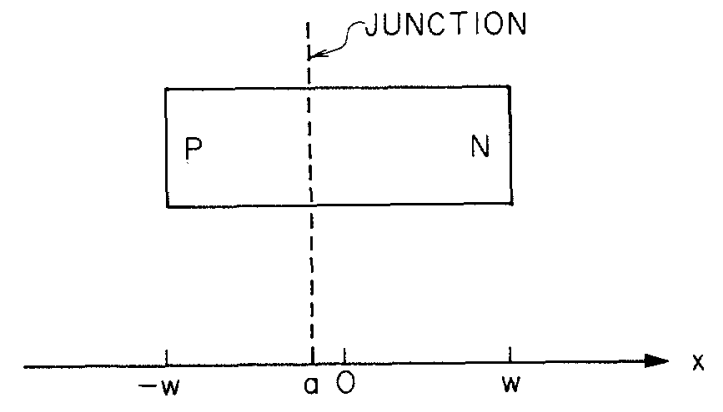

Fig. 1. Schematic diagram of the active waveguide with the lateral diffused junction at $x=a$.

sion coefficient, $n_{o m}$ is the minimal carrier density to achieve positive gain, $A$ is the optical gain constant, $\tau_{p h}$ is the photon lifetime, and $\tau_{s}$ is the spontaneous carrier lifetime. For simplicity, we neglect the contribution of spontaneous emission.

Given a photon distribution $S(x)$, one can solve for $\Delta(x)$ from (3) and (4), and the injection current density can be calculated from the resulting carriers density profile near $x=a$

$$
\begin{aligned}
J_{p}\left(a_{+}\right) & =-q D_{\mathrm{eff}}(x) \frac{d \Delta(x)}{d x} \\
J_{n}\left(a_{-}\right) & =+q D_{\mathrm{eff}}(x) \frac{d \Delta(x)}{d x} \\
J & =J_{p}\left(a_{+}\right)+J_{n}\left(a_{-}\right)
\end{aligned}
$$

where $J_{p}$ is the injected hole current density on the $n$ side, $J_{n}$ is the injected electron current density on the $p$ side, and $J$ is the total current density.

\section{Boundary Conditions}

Since the waveguide is surrounded by higher bandgap regions, we assume at $x=-W$ and $x=W$ that

$$
\begin{aligned}
& J_{p}(+W)=0 \\
& J_{n}(-W)=0
\end{aligned}
$$

Assuming thermal equilibrium is maintained throughout the waveguide, the Fletcher boundary condition [8] can be applied at the junction $x=a$ to relate the carrier densities on both sides:

$$
\begin{aligned}
& \frac{n_{n}+\Delta_{n}}{n_{p}+\Delta_{p}}=\frac{n_{n}}{n_{p}} e^{-q V / k T} \\
& \frac{p_{p}+\Delta_{p}}{p_{n}+\Delta_{n}}=\frac{p_{p}}{p_{n}} e^{-q V / k T} .
\end{aligned}
$$

In (8) and (9), $n_{n}$ and $p_{p}$ are the majority carrier densities, with $p_{n}$ and $n_{p}$ the corresponding minority carrier densities on the $n$ side and $p$ side, respectively, at thermal equilibrium. $\Delta_{n}$ is the excess carrier density on the $n$ side, and $\Delta_{p}$ is that on the $p$ side. $V$ is the applied forward bias voltage.

Two cases of interest are examined. In the first case, a p-n junction is located at $x=a$ with a high-level injection into both the $p$ and $n$ sides. From (8) and (9), we have at $x=a$

$$
\Delta_{n}\left(a_{+}\right)=\Delta_{p}\left(a_{-}\right) \text {. }
$$

Also, due to the high-level injection, $D_{\text {eff }}$ in (1) is equal to the ambipolar diffusion coefficient on both sides.

In the second case, a $\mathrm{p}^{+}-\mathrm{n}$ junction at $x=a$ produces a highlevel hole injection into the $n$ side, and a low-level electron injection into the $p$ side. At $x=a$ from (8) and (9) we have

$$
\left[\Delta_{n}\left(a_{+}\right)\right]^{2}=p_{p} \Delta_{p}\left(a_{-}\right) \text {. }
$$

In this case, $D_{\text {eff }}$ represents the ambipolar diffusion coefficient on the $n$ side, and $D_{\text {eff }}$ equals the diffusion coefficient of the $n$ type carriers on the $p$ side.

In the appendix, a self-consistent method is outlined for solving (3) and (4) for the carrier density profiles, with the photon profiles as given in (1), subject to the boundary conditions of (7), and the junction boundary condition given in (8) or (9).

\section{Discussion of the Calculated Results}

Case 1: Fig. 2 shows the calculated carrier profile in the case of a p-n junction, with high-level injection into both sides, assuming that lasing takes place predominantly in the fundamental mode $(m=0)$. The carrier density profiles are shown for different injection current densities. The $S_{0}$ 's given in Fig. 2 are in units of $1 /\left(A \tau_{s}\right)$ which takes the value of $1.3 \times$ $10^{14} \mathrm{~cm}^{-3}$. The value of $n_{\text {om }}$ is taken as $1.2 \times 10^{18} \mathrm{~cm}^{-3}$. $\tau_{s}$ and $\tau_{p h}$ are taken as 6 ns and $2 \mathrm{ps,} \mathrm{respectively.} \mathrm{The} \mathrm{width}$ of the waveguide is twice the ambipolar diffusion length $(\sim 2.6 \mu \mathrm{m})$, and $\xi$ is taken to be $W / 5$. Fig. 2 shows how the width (FWHM) of the carrier profile decreases as the injection current increases. An increase in the injection current causes an increase in the optical power, which results in a decrease in both the stimulated lifetime and hence the effective carrier diffusion length. As a result, the injected carrier profile narrows. To study the effect of the location of the junction on the threshold current density, the carrier density profiles with the junction located at $x=-3 W / 4$ are shown in Fig. 3. At a given injection level, the overlap of the gain distribution with the fundamental mode profile decreases as the junction is shifted away from the center. This results in a reduction in mode gain and thus an increase in the threshold current density. Also, as the junction approaches the $x=-W$ boundary of the waveguide, the situation resembles the one-sided injection situation in the TJS laser. As the portion of the $p$ side decreases, the injected electrons on the same side become more laterally confined, and, therefore, $J_{n}\left(a_{-}\right)$is reduced. The converse happens on the $n$ side and thus $J_{p}\left(a_{+}\right)$increases as the junction moves toward $x=-W$. In Fig. 4, threshold current density is plotted, for the fundamental mode $(m=0)$ and the first three higher order modes, as a function of the location of the junction. It is seen that, provided the junction is located near the center of the waveguide $(x=0)$, the fundamental mode has the lowest threshold current density and is thus favored. On the other hand, if the junction is located near the ends of the waveguide, higher order modes will be preferred. As the width of the waveguide is increased, a higher threshold current density is required, due to decreased carrier confinement in the lateral direction. This also causes an increase in the width of the lossy regions where $\Delta(x)$ is less than $n_{o m}$. Hence, the carrier profile tends to be more sharply peaked as the width of the 


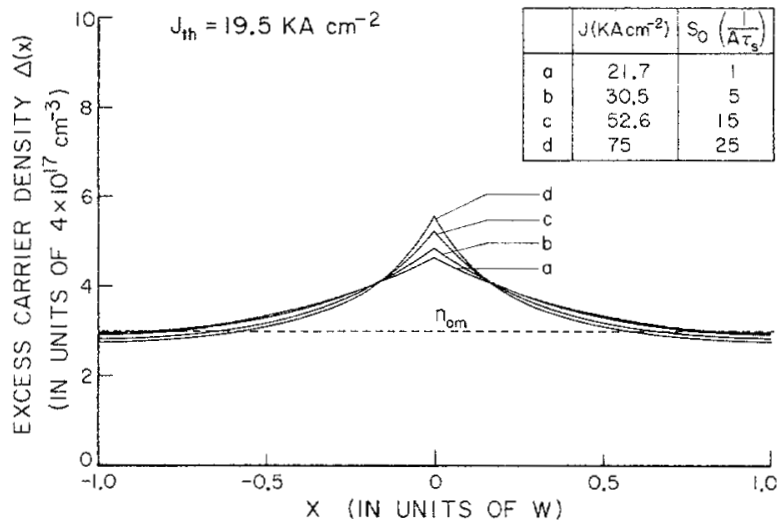

Fig. 2. Excess carrier density profiles for a p-n junction at different injection current densities. The junction is located at $x=0, S_{0}$ 's are in units of $1 /\left(A \tau_{s}\right)\left(1.3 \times 10^{14} \mathrm{~cm}^{-3}\right), W$ is $2.6 \mu \mathrm{m}$. Threshold current density is $19.5 \mathrm{kA} \cdot \mathrm{cm}^{-2}$. The dashed line shows the $n_{o m}$.

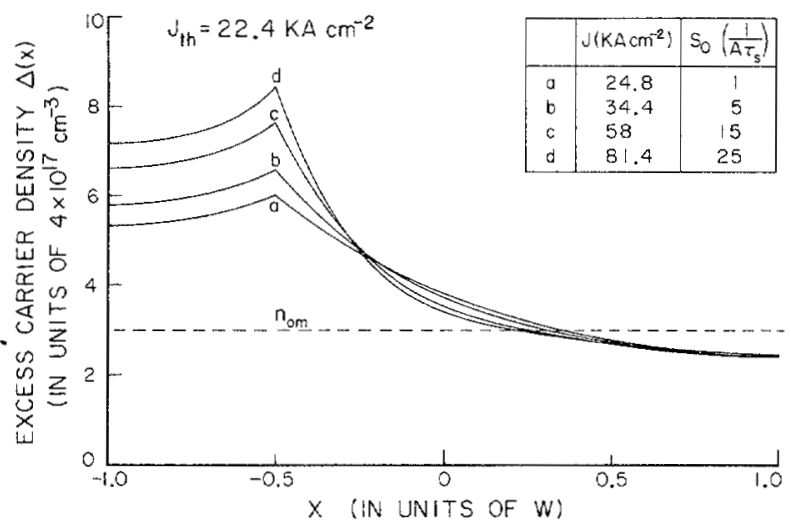

Fig. 3. Excess carrier density profiles for a p-n junction at different injection current densities, with junction at $x=-3 W / 4$. Threshold current density is $22.4 \mathrm{kA} \cdot \mathrm{cm}^{-2}$.

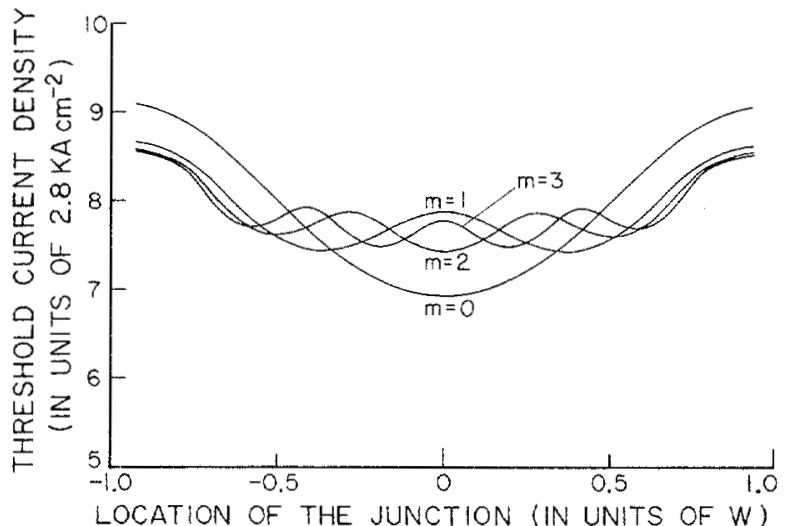

Fig. 4. Threshold current density for a p-n junction, versus the location of the junction, with the laser lases predominantly in the $m$ th mode.

waveguide increases. In Fig. 5, we plot the threshold current density of a laser with the junction located in the middle of the waveguide, versus the width of the waveguide. The laser is assumed to operate predominantly in the fundamental mode. The relative degree to which various lateral modes are preferred or discriminated against can be quantitatively expressed by the modal gain $\gamma_{m}$.

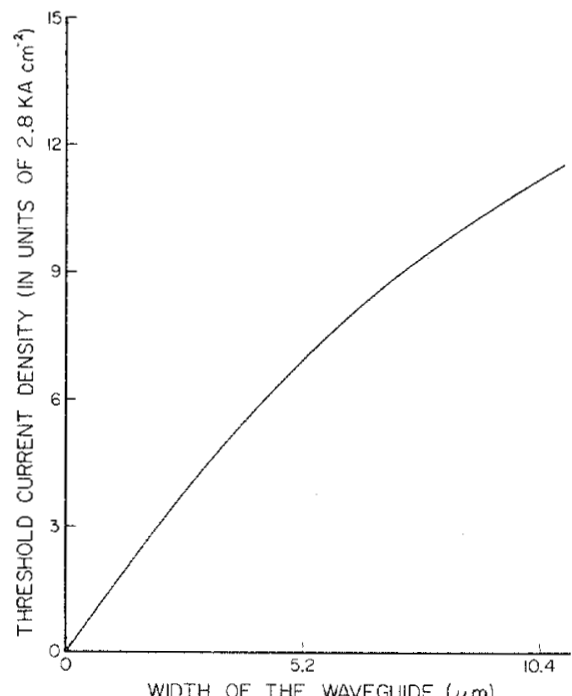

Fig. 5. Threshold current density for the fundamental mode, in the case of a $p-n$ junction, as a function of the width of the waveguide $(2 W)$

$$
\gamma_{m}=\frac{n_{0}}{c} \times \frac{\int_{-W}^{+W} A \Delta(x) S_{m}(x) d x}{\int_{-W}^{+W} S_{m}(x) d x}
$$

where $n_{0}$ is the index of refraction of the region, $\Delta(x)$ is the excess carrier density profile, and $S_{m}(x)$ is the intensity profile of the $m$ th mode. In principle, $\Delta(x)$ can be obtained from (3) and (4) only when the lasing amplitudes of all the transverse modes are known. As a first order approximation, with the diffused junction located at the center of the waveguide, we can assume that lasing occurs predominantly in the fundamental mode and proceed to solve for $\Delta(x)$ from (3) and (4). The resulting carrier distribution $\Delta(x)$ can then be used in (12) to calculate the gain $\gamma_{m}$ of the higher order modes. The values of the $\gamma_{m}$ thus obtained will be an indication of the stability of the lasing in the fundamental mode. The results are shown in Fig. 6 , in which the modal gain $\gamma_{m}$ is plotted as a function of the injection current density $J$ for different modes. From the figure, it is observed that the first order mode $(m=1)$ and the third order mode $(m=3)$ are discriminated against as $J$ increases while the second order mode $(m=2)$ is not much affected. This results in the increasing tendency of the fundamental mode operation as the injection current is increased. This behavior has not been observed in the other laser structures. In some structures, such as the channeled substrate laser [9], gains of higher order modes converge to that of the fundamental mode as the injection level is raised.

Case 2: In the case of a $\mathrm{p}^{+}-\mathrm{n}$ junction, the same parameters are used as in Case 1 above, except that the ratio of the diffusion coefficient on the $p$ side to that on the $n$ side is taken to be 2.25. The acceptor concentration $N_{A}$ takes the value of $4 \times 10^{18} \mathrm{~cm}^{-3}$. Again, assuming lasing in the fundamental mode $(m=0)$, the carrier density profiles shown in Fig. 7 are for different injection current densities. Since the ambipolar diffusion length is longer on the $p$ side in the present case 


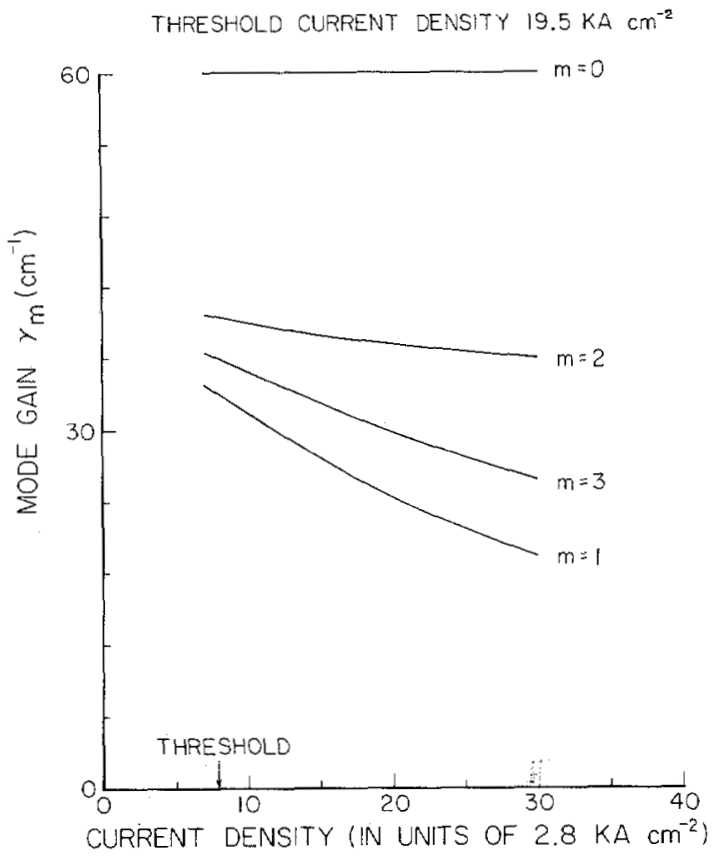

Fig. 6. Modal gains for the first four modes, in the case of a $p-n$ junction, as a function of the injection current density. The diffused junction is located at $x=0$.

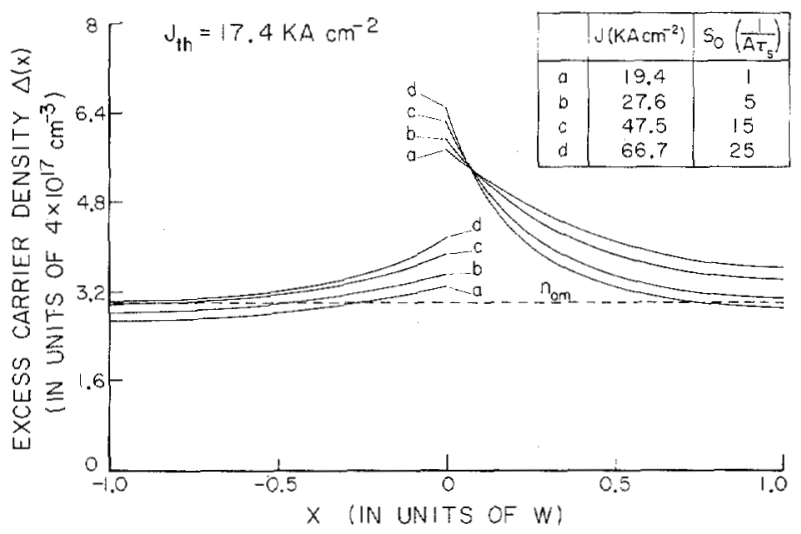

Fig. 7. Excess carrier density profiles for a $\mathrm{p}^{+}-\mathrm{n}$ junction at different injection current densities, with junction located at $x=0$. Threshold current density is $17.4 \mathrm{kA} \cdot \mathrm{cm}^{-2}$.

compared to that in the case of a $p-n$ junction, the electron is more confined on the $p$ side in the case of a $\mathrm{p}^{+}-\mathrm{n}$ junction. This results in a reduction of threshold current density, as mentioned in Case 1. In effect, an increase in diffusion length is equivalent to a decrease in the effective width of the waveguide. Furthermore, the modal behavior in the present case can be understood from the reduction of the effective waveguide width on the $p$ side. Thus, when the junction is on the $x<0$ side, the situation is closer to that shown in Fig. 3 ; and when the junction is on the $x>0$ side, the situation is like that of a p-n junction, with the width of the waveguide on the $p$ side replaced by the effective width. Therefore, at equal distance from the middle, the threshold will be higher when the junction is on the $x<0$ side than when it is on the $x>0$ side. Other features, such as the threshold current density as a function of the waveguide width, the mode discriminating behavior, and the mode stabilization behavior, will be similar to that in Case 1 above. This is expected, because as the injection level increases, the two cases become increasingly similar.

\section{Validity of the Above Analysis}

The validity of the above results rests on the assumption, stated in Section II, that the spatial mode is index-guided and is independent of the injection level. Under high-level injection, however, the localization of carriers near the junction provides a gain-guiding mechanism so that a combination of index and gain guiding may take place. Gain guiding may thus become increasingly dominant at very high-level injection and in the case of a very wide waveguide. The above analysis is valid when the width of the optical mode guided by the gain profile is much wider than that guided by the real-index waveguide, which is approximately equal to half of the width of the waveguide. For a rough estimate of the width of the gainguided mode, the exact shape of the gain profile is not important and one can use the result for the approximate fundamental width $x_{m}$ in the case of a Gaussian gain profile [10].

$$
\begin{aligned}
S(x) & =S_{0} e^{-x^{2} / L^{2}} \\
x_{m} & =2\left(\frac{\lambda L}{2 \pi}\right)^{1 / 2}\left(\frac{S_{0} \lambda n}{\pi}\right)^{-1 / 4}
\end{aligned}
$$

where $\lambda$ is the free space wavelength, $L$ is the diffusion length, $S(x)$ and $S_{0}$ are in $\mathrm{cm}^{-1}$, and $n$ is the effective refractive index of the medium. One can use the values of the peak gain and half width of the gain profile obtained in the above section in (13) and determine $x_{m}$. The results described in the preceding section cease to be valid when $x_{m} \leqslant W / 2$. If one limits the widths of the waveguide to less than $5 \mu \mathrm{m}$ and assuming diffusion length is $2 \mu \mathrm{m}$, one can estimate from (13) that index guiding is the dominant mechanism for a peak gain as high as $1000 \mathrm{~cm}^{-1}$, which corresponds to a pump current $J$ density of about $90 \mathrm{kA} \cdot \mathrm{cm}^{-2}$. This sets an upper limit on the range of validity of the above analysis.

In the above analysis, we have for simplicity neglected the effect of the evanescent waves outside the guide. Since the fundamental mode is best confined to the active region, the effect of the inclusion of the evanescent waves would only improve further the calculated stabilization of the fundamental mode.

\section{COMParisons With EXPERIMENTS}

The schematic diagram of the groove $\mathrm{InGaAs} \mathrm{P} / \mathrm{In} \mathrm{P}$ transverse junction laser is shown in Fig. 8. Three LPE layers: n-InP (undoped, with background electron concentration level at 4-9 $\times 10^{16} \mathrm{~cm}^{-3}$ ), n-InGaAsP (undoped), and n-InP ( $\mathrm{Sn}$ doped, $2 \times 10^{18} \mathrm{~cm}^{-3}$ ) are grown successively on a grooved and masked semi-insulating INP substrate [11]. This is followed by a lateral $\mathrm{Zn}$ diffusion. Depending on the depth of diffusion, the resulting junction will be a $\mathrm{p}-\mathrm{n}$ junction or a $\mathrm{p}^{+}-\mathrm{n}$ junction. The width of the active region is $3-5 \mu \mathrm{m}$, and the thickness is $\sim 0.5-1 \mu \mathrm{m}$ at the center of the active region. The lasers are tested with 50 ns current pulses having a repetition rate of $1 \mathrm{kHz}$. Stable lateral mode operation is achieved up to 45 times threshold current, when the $\mathrm{Zn}$ diffusion front is placed somewhere near the middle of the active region, consistent 


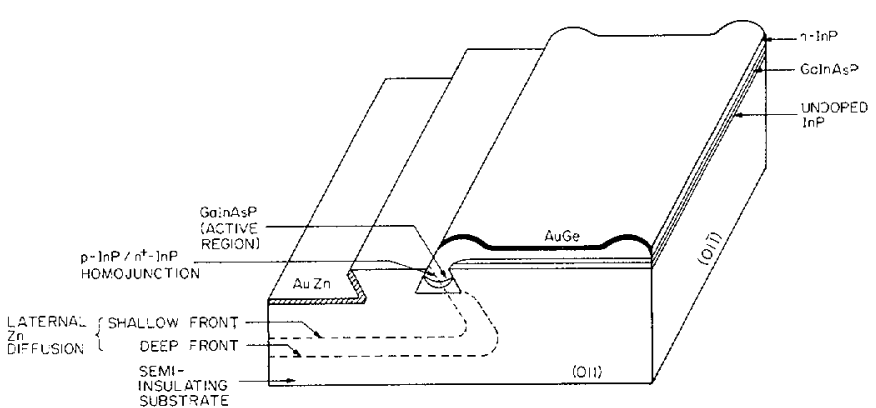

Fig. 8. Schematic diagram of the groove transverse junction laser on semi-insulating InP. Width of the active region is $3-5 \mu \mathrm{m}$.

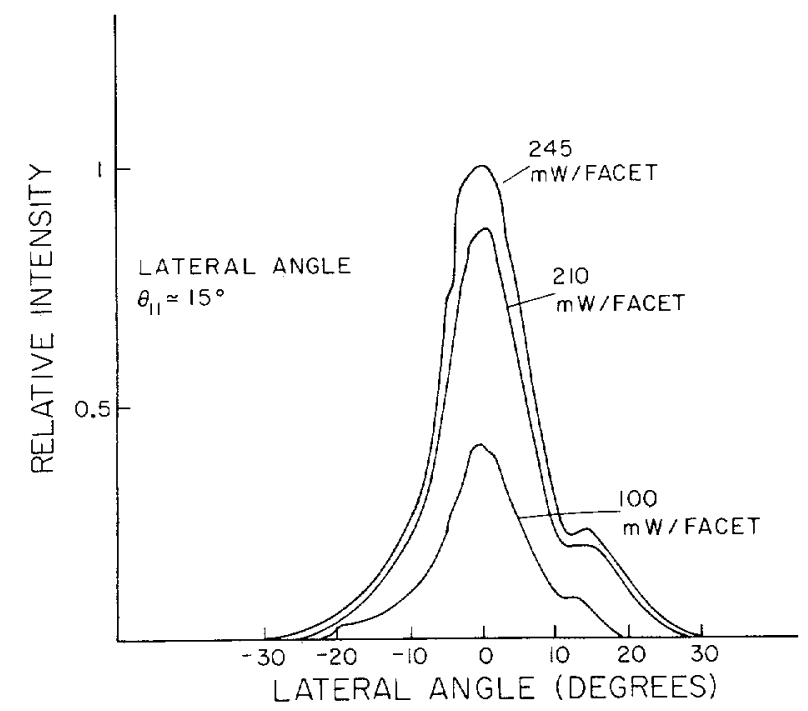

Fig. 9. Far field pattern of one groove laser, with lateral diffused junction in the middle of the active region, at different injection currents.

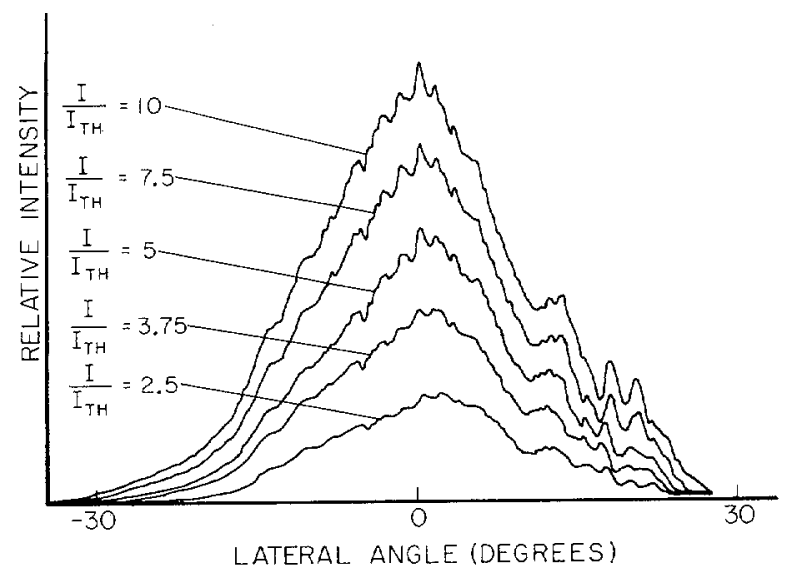

Fig. 10. Far field pattern of another groove laser, with lateral diffused junction in the middle.

with the calculations above. Figs. 9 and 10 show typical far field patterns of the lasers with diffusion front in the middle. The lasers are operated with current pulses having a width of $50 \mathrm{~ns}$ and a repetition rate of $1 \mathrm{kHz}$. The fact that the spatial mode profile does not change significantly as the power output is increased is an indication that the laser is operating predominantly in the fundamental spatial mode at all power levels. However, as can be seen in Figs. 9 and 10, there are irregularities in the far field patterns which are probably due to the

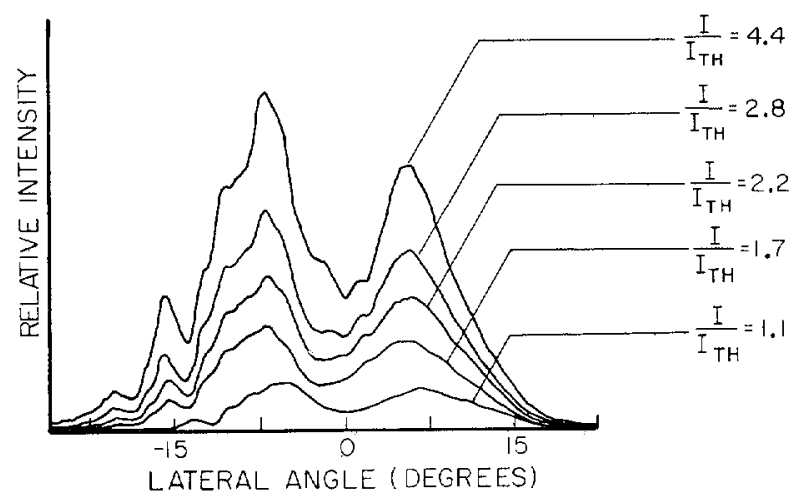

Fig. 11. The spectrum of the same laser shown in Fig. 10 at different injection currents.

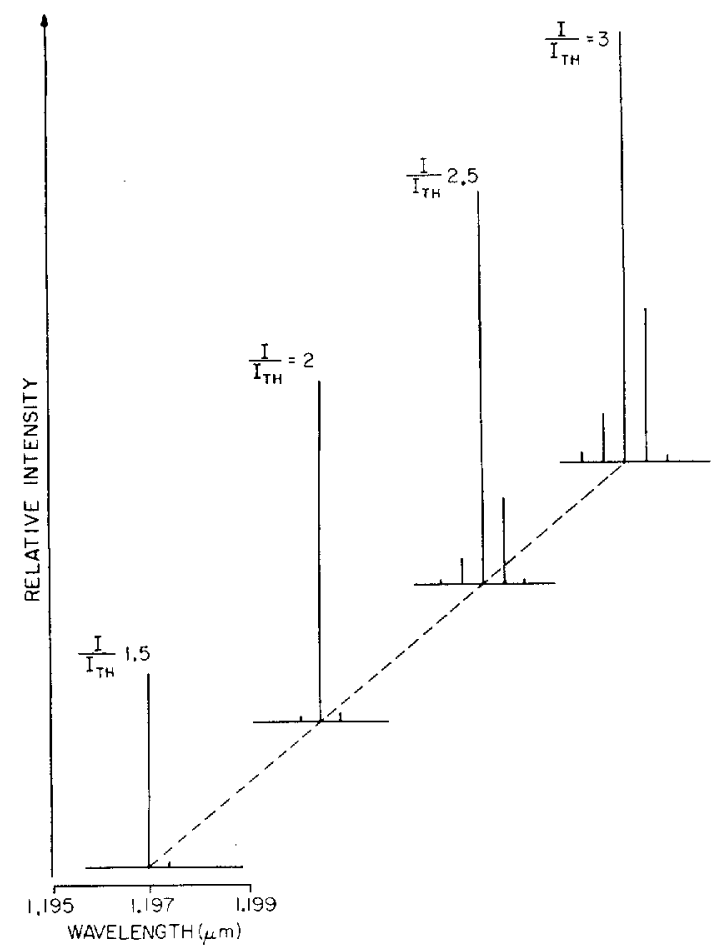

Fig. 12. A typical far field pattern of the groove laser, with lateral diffused junction touching the active region, at different injection currents.

scattering of light by the etched sidewalls. Most of the lasers with junction at the middle of the waveguide operate in a single longitudinal mode up to 1.2-1.4 times threshold current; however, for some of the lasers, predominantly single longitudinal mode operation is maintained up to 2-3 times threshold current. The spectra shown in Fig. 11, which illustrate single longitudinal mode operation up to 2 times threshold current, belong to the same laser whose far field is shown in Fig. 10. When the diffusion front is near the edge of the active region, higher order transverse modes are observed. Fig. 12 shows a typical far field pattern, taken under the same testing condition, of the laser with diffusion front just touching the active region. It is also observed that an increase in the width of the active region will result in a slight increase in the threshold current density, in qualitative agreement with the model described above. 


\section{Conclusion}

In conclusion, we have demonstrated the gain stabilizing behavior of a waveguide with lateral carrier injection. The finite width of the waveguide, as compared to the carrier diffusion length, together with the boundary conditions, result in the confinement of carriers within a small region about the lateral junction. As the current injection increases, the optical power increases, and the gain profile becomes progressively narrower due to the decrease of the stimulated lifetime. Stabilization for the fundamental mode is obtained when the transverse junction is placed near the middle of the waveguide. These results are in good agreement with experimental observations in which the groove GaInAsP/InP lasers show a remarkable insistence on operating in the fundamental lateral mode when the lateral diffused junction is placed at the middle of the active region. The model and analysis developed in this work can be used to predict the modal behaviors in similar structures.

\section{APPENDIX}

We divide the waveguide into $h$ regions, with the width $w_{k}$ in the $k$ th region where $k=1, \cdots, h$. Next, taking $S(x)$ as given by (1) as a constant in each region, we can solve (3) and (4) for the excess carrier density. Thus, in the $k$ th region

$$
\Delta_{k}\left(x_{k}\right)=A_{k} e^{-x_{k} / L_{k}}+B_{k} e^{x_{k} / L_{k}}+C_{k} \quad 0 \leqslant x_{k} \leqslant w_{k}
$$

where

$$
\begin{aligned}
L_{k} & =\frac{L}{\sqrt{1+S_{k} A \tau_{s}}} \\
C_{k} & =\frac{A n_{o m} S_{k} L_{k}^{2}}{D_{\text {eff }}} .
\end{aligned}
$$

$L$ is the diffusion length in that region, and $L_{k}$ is the effective diffusion length in the presence of the photon intensity profile.

The coefficients $A_{k}, B_{k}$ are determined by matching boundary conditions at $x_{k}=w_{k}$.

$$
\begin{aligned}
A_{k}= & \frac{e^{-w_{k} / L_{k}}}{2}\left(A_{k+1}\left(1-\frac{L_{k}}{L_{k+1}}\right)\right. \\
& +B_{k+1}\left(1+\frac{L_{k}}{L_{k+1}}\right)+C_{k+1}-C_{k} \\
B_{k}= & \frac{e^{w_{k} / L_{k}}}{2}\left(A_{k+1}\left(1+\frac{L_{k}}{L_{k+1}}\right)\right. \\
& +B_{k+1}\left(1-\frac{L_{k}}{L_{k+1}}\right)+C_{k+1}-C_{k} \\
A_{h}= & B_{h} e^{-2 w_{h} / L_{h}} \\
A_{1}= & B_{1}
\end{aligned}
$$

Defining $B_{h}=K_{1}, B_{1}=K_{2}$, using the recurrence relations (A-2) and (A-3), coefficients $A_{h}, A_{h-1}, B_{h-1}, \cdots$ can be calculated as a linear function of $K_{1}$. Similarly, $A_{1}, A_{2}, B_{2}$ can be calculated as a linear function of $K_{2}$. By matching the junction boundary condition at $x=a$, which for the case of a $\mathrm{p}-\mathrm{n}$ junction, (as given by 10 ) gives

$$
K_{2}=c_{1} K_{1}+c_{0}
$$

and for the case of a $\mathrm{p}^{+}-\mathrm{n}$ junction, (12) results in

$$
K_{2}=d_{2} K_{1}^{2}+d_{1} K_{1}+d_{0}
$$

where $c_{1}, c_{0}, d_{2}, d_{1}$, and $d_{0}$ are determined from computation. Once $K_{1}$ is known, all the $A$ 's and $B$ 's can be determined. To find $K_{1}$, we go back to $(1)$, which now takes the form

$$
\begin{aligned}
\sum_{k=0}^{h} S_{k} w_{k}\left(1-C_{k}+n_{o m}\right)= & \sum_{k=0}^{h} S_{k} L_{k}\left(A_{k}\left(1-e^{-w_{k} / L_{k}}\right)\right. \\
& \left.+B_{k}\left(e^{w_{k} / L_{k}}-1\right)\right)
\end{aligned}
$$

The left side of (A-8) is constant once $S_{0}$ is given, the right side is a linear function of $K_{1}$ if (A-6) is used, or a quadratic function of $K_{1}$ if (A-7) is used. In both cases $K_{1}$ can be found and thus the carrier density profiles can be computed.

\section{REFERENCES}

[1] S. Wang, C. Y. Chen, A. S. Liao, and L. Figueroa, "Control of mode behavior in semiconductor lasers," IEEE I. Quantum Electron., vol. QE-17, pp. 453-468, 1981.

[2] T. R. Chen, U. Koren, K. L. Yu, K. Y. Lau, L. C. Chiu, A. Hasson, S. Margalit, and A. Yariv, "High power, single-mode operation of an InGaAsP/InP laser with a grooved transverse junction using gain stabilization," Appl. Phys. Lett., vol. 41, pp. 225-228, 1982.

[3] D. C. Krupka, "Selection of modes perpendicular to the junction plane in GaAs-large cavity double-heterostructure lasers," IEEE $J$. Quantum Electron., vol. QE-11, pp. 390-400, 1975.

[4] T. L. Paoli, B. W. Habbi, and B. I. Miller, "Zero-order transverse mode operation of $\mathrm{GaAs}$ double-heterostructure lasers with thick waveguides," J. Appl. Phys., vol. 44, pp. 1276-1281, 1973.

[5] W. W. Anderson, "Mode confinement and gain in injection laser," IEEE J. Quantum Electron., vol. QE-1, pp. 228-236, 1965.

[6] J. K. Butler, "Theory of transverse cavity mode selection in homojunction and heterojunction semiconductor laser diodes," J. Appl. Phys., vol. 42, pp. 4447-4457, 1971.

[7] J. K. Butler and H. Kressel, "Transverse mode selection in injection laser with widely spaced heterojunction," $J$. Appl. Phys., vol. 43, pp. 3403-3411, 1972.

[8] N. H. Fletcher, "The high current limit for semiconductor junction devices," Proc. IRE, vol. 45, pp. 862-872, 1957.

[9] D. P. Wilt and A. Yariv, "A self consistent static model of the double heterostructure laser," IEEE J. Quantum Electron., vol. QE-17, pp. 1941-1949, 1981.

[10] K. Y. Lau and A. Yariv, "Transverse-modal behavior of a transverse junction stripe laser exhibited by a short electrical pulse," J. Appl. Phys., vol. 52, pp. 641-645, 1981.

[11] K. L. Yu, U. Koren, T. R. Chen, P. C. Chen, and A. Yariv, "Groove GaInAsP laser on semi-insulating InP," Electron. Lett., vol. 17, pp. $790-791,1981$.

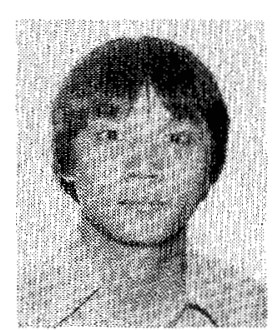

K. L. Yu received the B.S. degree in physics and the M.S. degree in applied physics from the California Institute of Technology, Pasadena, in 1979.

Currently he is working on a doctoral dissertation in the field of optoelectronic devices for optical communication at the California Institute of Technology.

Mr. Yu is a member of the American Physical Society and Tau Beta Pi. 


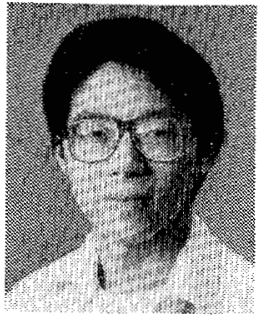

Kam Y. Lau (M'78) was born in Hong Kong in October 1956 . He received simultaneously the B.S. and M.S. degrees in electrical engineering in June 1978, and the Ph.D. degree in electrical engineering in June 1981, all from the California Institute of Technology, Pasadena.

He worked at Bell Laboratories, Holmdel, NJ, on optical modulators during the summers of 1977 and 1978. While in graduate school, he worked part time at the Jet Propulsion Laboratory, Pasadena, CA, where he was responsible for development of fiber optic systems for specialized applications in the NASA Deep Space Network. He received a Distinguished Achievement Award from NASA in 1981. He was a Research Fellow at the California Institute of Technology from June 1981 until he joined the Ortel Corporation, Alhambra, CA, in October 1981, where he is currently continuing work on semiconductor lasers and related technologies.

Dr. Lau is a member of the Optical Society of America.

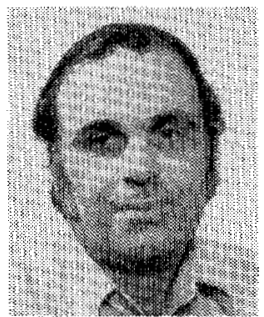

Shlomo Margalit was born in Haifa, Israel, on July 29,1941 . He received the B.Sc., M.Sc., and D.Sc. degrees from the Technion-Israel Institute of Technology, Haifa, Israel, in 1965, 1968 , and 1971 , respectively.

Since 1971 he has been a Faculty Member of the Department of Electrical Engineering, Technion-Israel Institute of Technology. In 1980 he became an Associate Professor. During the academic years 1977-1978 and 1981-1982 he was a Visiting Associate at the California Institute of Technology, Pasadena. His research interests are in the field of semiconductor devices.

Uziel Koren was born in Jerusalem, Israel, in 1946. He received the B.Sc. degree in physics and mathematics and the M.Sc. degree in physics from the Hebrew University, Jerusalem, Israel, and the Ph.D. degree from Weizmann Institute of Science, Rehovot, Israel, in 1978.

He was a Research Fellow at the Weizmann Institute of Science until 1980 , where he was engaged in research on infrared detectors and systems. Currently, he is a Research Associate at the California Institute of Technology, Pasadena, where he is working on semiconductor optoelectronic devices.

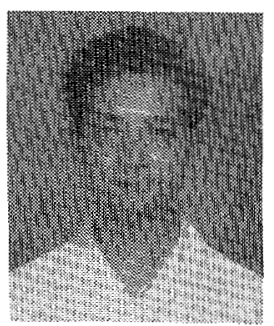

T. R. Chen was born in January 1940. He graduated from Wuhan University, Wuhan, China, in 1962.

He joined the faculty of Chengdu Institute of Radio Engineering, where he conducted research in gas lasers, optical communication, and cavity theory. Since 1980 he has been a Visiting Associate at the California Institute of Technology, Pasadena. His interests include semiconductor lasers, optoelectronic integration, and optical communication.

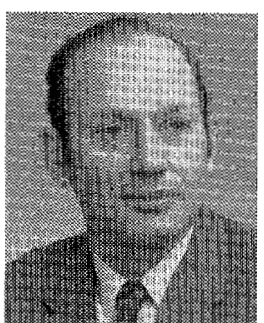

Amnon Yariv (S'56-M'59-F'70) was born in Tel-Aviv, Israel, on April 13, 1930. He received the B.S., the M.S., and the Ph.D. degrees, all in electrical engineering, from the University of California, Berkeley, in 1954, 1956, and 1958, respectively.

In 1959 he went to Bell Laboratories, Murray Hill, NJ, where he joined the early stages of the laser effort. In 1964 he joined the California Institute of Technology, Pasadena, as an Associate Professor Electrical Engineering, and in 1966 he became a Professor. In 1980 he became the Thomas G. Myers Professor of Electrical Engineering and Applied Physics. On the technical side, he took part (with various co-workers) in the discovery of a number of early solid-state laser systems, the formulation of the theory of parametric quantum noise and the prediction of parametric fluorescence, the invention of the technique of mode-locked ultrashort-pulse lasers and FM lasers, the introduction of GaAs and CdTe as infrared electrooptic and window materials, proposing and demonstrating semiconductor based integrated optics technology, and pioneering the field of phase conjugate optics. His present research efforts are in the areas of nonlinear optics, recombination mechanisms in semiconductors, semiconductor lasers, and integrated optics, especially the problem of monolithic integration of transistors, injection lasers, and detectors for high-frequency applications. He authored or coauthored some 190 papers in professional journals, as well as two books: Quantum Electronics (New York: Wiley 1967, 1975) and Introduction to Optical Electronics (New York: Holt, Rinehart, and Winston, 1971, 1976). He is also the Associate Editor of Optics Communications and the Journal of Applied Physics, and was previously Associate Editor of the IEEE JoURnal of QUANTUM ELECTRONICS.

Dr. Yariv is a member of the American Physical Society, Phi Beta Kappa, and the National Academy of Engineering, and a Fellow of the Optical Society of America. He is a consultant to the Hughes Aircraft Research Laboratories and is the recipient of the 1980 Quantum Electronics Award of the IEEE. 